\title{
Optimization of Comminution Circuit Throughput and Product Size Distribution by Simulation and Control Quarterly Technical Progress Report
}

Report Period Start Date:

Report Period End Date:

Authors:

Date of Issue:

DOE Award Number:
April 01, 2004

June 30, 2004

H.J. Walqui - Graduate Student

T. C. Eisele - Engineer/Scientist

S. K. Kawatra - Principal Investigator

July 2004

DE-FC26-01NT41062

Submitting Organization

Department of Chemical Engineering

Michigan Technological University

1400 Townsend Drive

Houghton, MI 49931 - 1295 


\section{DISCLAIMER:}

This report was prepared as an account work sponsored by an agency of the United States Government. Neither the United States Government nor any agency thereof, nor any of their employees, makes any warranty, express or implied, or assumes any legal liability or responsibility for the accuracy, completeness, or usefulness of any information, apparatus, product, or process disclosed, or represents that its use would not infringe privately

owned rights. Reference herein to any specific commercial product, process, or service by trade name, trademark, manufacturer, or otherwise does not necessarily constitute or imply its endorsement, recommendation, or favoring by the United States Government or any agency thereof. The views and opinions of authors expressed herein do not necessarily state or reflect those of the United States Government or any agency thereof. 


\begin{abstract}
The goal of this project is to improve energy efficiency of industrial crushing and grinding operations (comminution). Mathematical models of the comminution process are being used to study methods for optimizing the product size distribution, so that the amount of excessively fine material produced can be minimized. The goal is to save energy by reducing the amount of material that is ground below the target size, while simultaneously reducing the quantity of materials wasted as "slimes" that are too fine to be useful. This is being accomplished by mathematical modeling of the grinding circuits to determine how to correct this problem. It has been determined that, for mixtures of approximately equal quantities of high-density minerals (such as iron oxides) and lowdensity minerals (such as quartz), existing hydrocyclone models fail to accurately predict the hydrocyclone behavior. Since the hydrocyclone is the key unit controlling the particle size, an accurate model of these units is required and is being fully developed.

Experimental work has demonstrated that the previous models are inaccurate due to incorrect assumptions concerning the change in hydrocyclone cut size as a function of changing particle density.
\end{abstract}


Table of Contents

Introduction $\quad 5$

Executive Summary

$\begin{array}{ll}\text { Experimental } & 7\end{array}$

Results and Discussion $\quad 8$

$\begin{array}{ll}\text { Conclusions } & 9\end{array}$

$\begin{array}{ll}\text { References } & 10\end{array}$

List of Tables and Graphical Materials

Figure 1: Comparison between experimentally-determined efficiency curves, and efficiency curves predicted for this material by Plitt's model

Figure 2: Model fit with experimental data after inclusion of a shape correction term. 


\section{Introduction}

While crushing and grinding (comminution) of various feedstocks is a critical operation in mining, as well as in a range of other industries, it is both energy-intensive and expensive, with tremendous room for improvement. A neglected route in optimizing the comminution process is the minimizing of overgrinding. Since grinding particles to finer than the target size both wastes energy and produces unusable product, such overgrinding must be minimized in order to improve energy efficiency. The objective of this project is therefore to sample and simulate a full-scale iron ore processing plant to determine methods for increasing grinding circuit energy efficiency by minimizing overgrinding.

Plant sampling and analysis has demonstrated that the largest single source of overgrinding in the industrial process is the return of high-density material to the grinding process by hydrocyclones. The particles of high-density iron oxides that are near the cut size are fully liberated and do not require further comminution, but they are returned to the mill rather than being removed as final product. A fundamental redesign of the comminution circuit is therefore needed to deal with these intermediate-size dense particles. It has also been determined, from extensive experimental work, that the existing hydrocyclone models are inadequate for situations where the slurry contains a mixture of very fine high-density and low-density particles. Work is therefore proceeding to develop a hydrocyclone model that can be used with such mixtures.

\section{Executive Summary}

The goal of a comminution circuit is to grind particles to their liberation size, so that the valuable minerals are completely broken free from the gangue minerals. For the best grinding efficiency, all particles must be ground only to the liberation size, and no finer. To accomplish this, grinding mills are generally operated in closed circuit with a sizing device, such as a screen or a hydrocyclone, so that as soon as particles reach the target size they are removed from the circuit by the sizing device. In most grinding circuits, the hydrocyclone is the preferred sizing device because it can classify very fine particles, and it is rugged and inexpensive to operate. Screens for sizing very fine particles would be theoretically superior to hydrocyclones for this application, but they are much larger and at fine particle sizes they tend to be very high-maintenance items.

When samples were collected from the plant, it was determined that approximately $35 \%$ of the hydrocyclone underflow that is returned to the pebble mills is liberated magnetite that does not need to be ground further. Coarsening the separation by the hydrocyclone would prevent this magnetite from being overground, but would also result in an unacceptable amount of locked quartz/magnetite particles leaving the comminution circuit.

In the course of analyses of samples collected from hydrocyclones in an operating plant, the products were chemically analyzed by size and used to calculate the individual efficiency curves for just the (high density) magnetite and the (low density) quartz, as 
well as the overall efficiency curve for the mixture. However, when these curves were modeled using the standard hydrocyclone models available in the literature, it was found that the measured individual hydrocyclone efficiency curves for iron oxides and quartz did not match the curves, as can be seen in Figure 1.

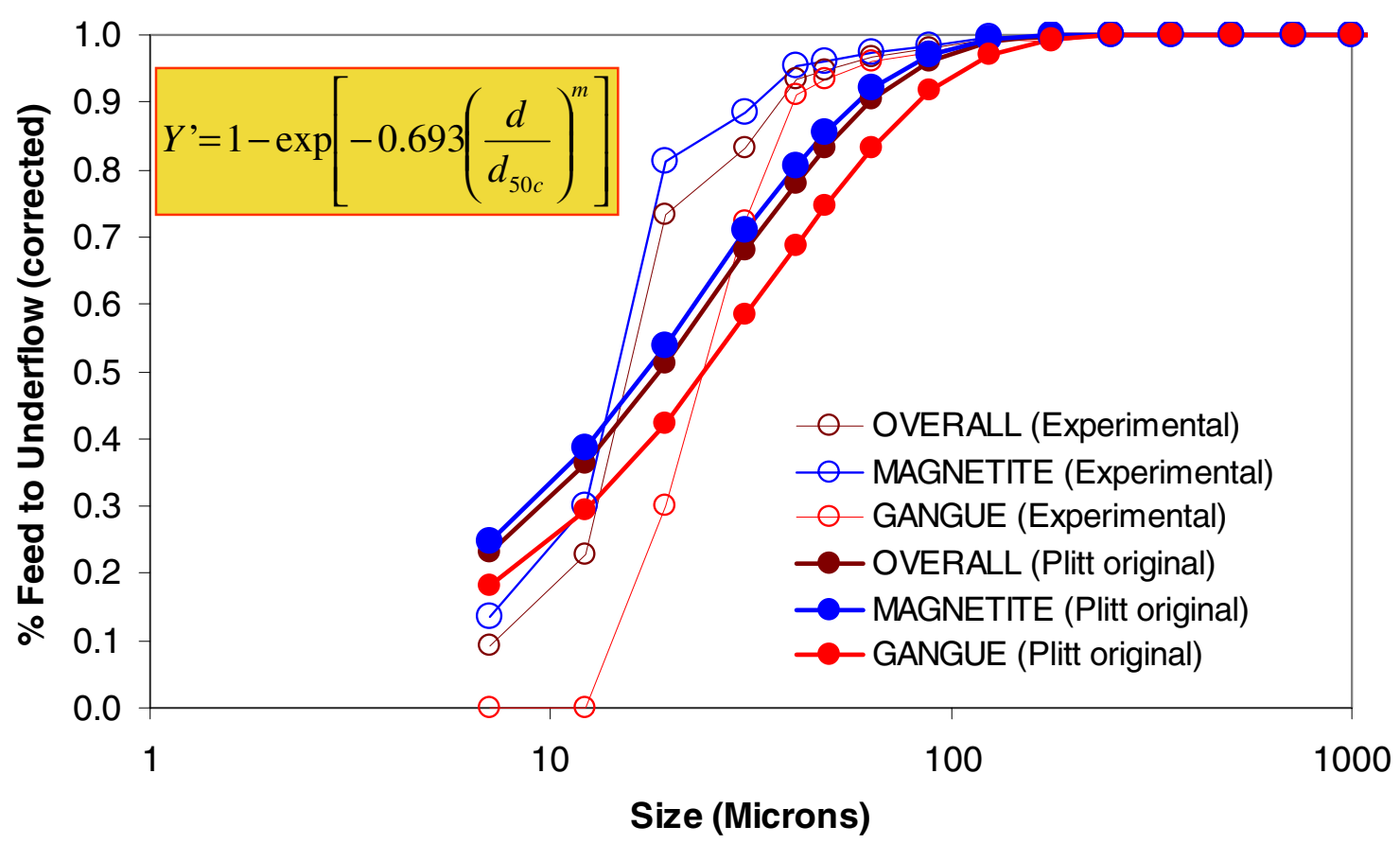

Figure 1: Comparison between experimentally-determined efficiency curves, and efficiency curves predicted for this material by Plitt's model (Plitt, 1976). This model both failed to match the shape of the experimental curve, and showed an insufficient spacing beteween the high-density and low-density curves.

Significant changes were therefore necessary to the hydrocyclone model. The first was the introduction of a shape correction term, that would allow the model curves to match the curvatures of the experimental curves. The results of this correction are shown in Figure 2, where it can be seen that the model predictions much more closely match the shape of the experimental curves. The correction allows deviation from the ideal "S" shaped efficiency curve by adjustment of the parameters B and C (Whiten, 2004).

The second change needed is to correct for the insufficient spacing between the magnetite and quartz curves. In all hydrocyclone models to date, it has been assumed that the cut size (d50) for the hydrocyclone is proportional to the square root of the difference between the particle density and the liquid density. This is expressed by the value of the constant $\mathrm{K}_{2}$, which has been set at 0.5 in all past models. From the experimental data, this assumption appears to be incorrect. Studies are therefore underway to determine whether the difference between theory and experiment is due to variations in the slurry density, or whether it is due to the true value of $\mathrm{K}_{2}$ being different from 0.5 . 


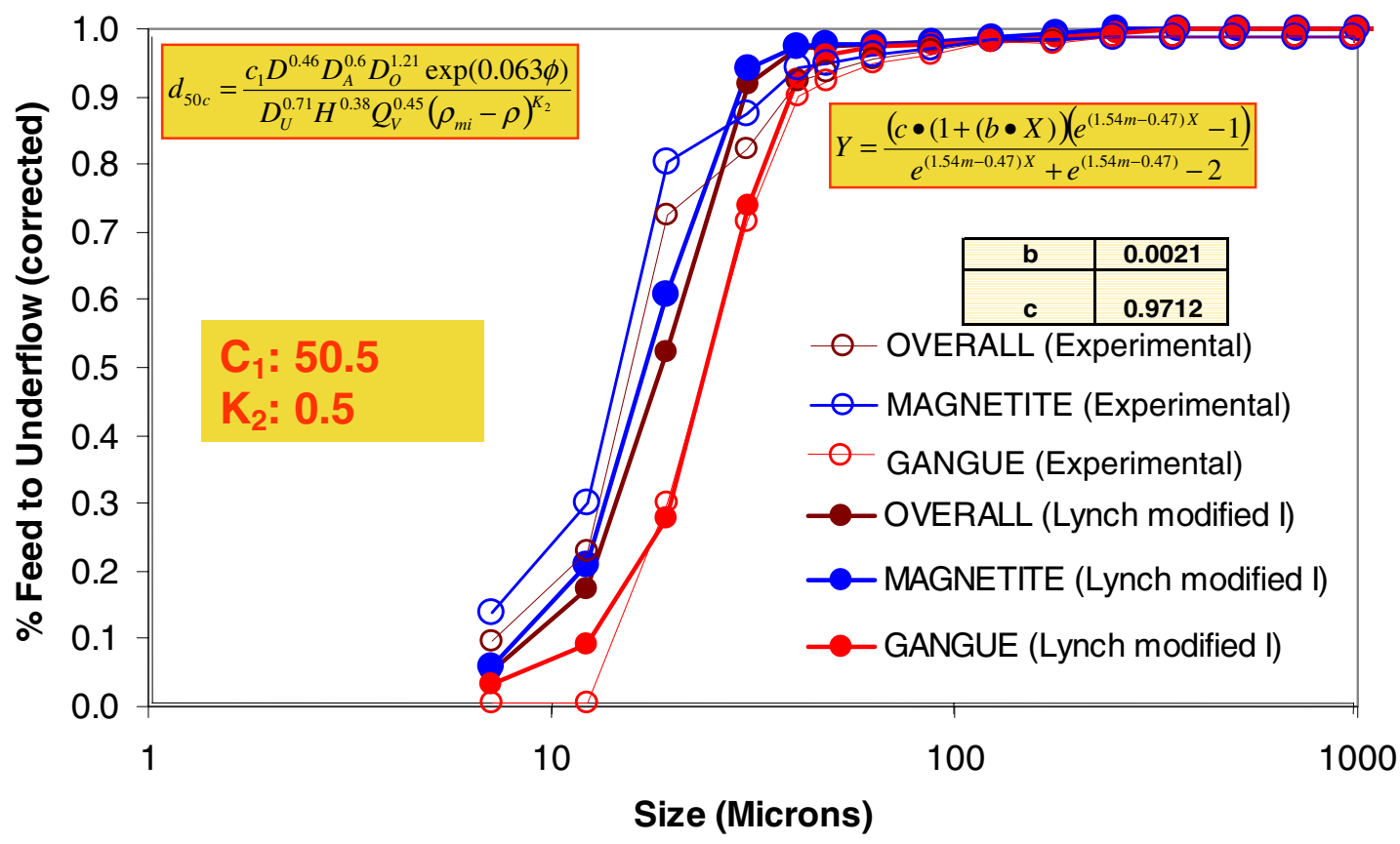

Figure 2: Model fit with experimental data after inclusion of a shape correction term. The match to the shape of the cuves is much improved, particularly for the gangue (quartz) curve, but there is still insufficient separation between the high-density (magnetite) and the low-density (quartz) curves.

\section{Experimental}

Previously in this project, samples had been collected from hydrocyclones in an operating iron ore concentrator. The cyclones were operated in a bank of 14 units, each $34.9 \mathrm{~cm}$ in internal diameter, with $13.35 \mathrm{~cm}$ vortex finers and $6.35 \mathrm{~cm}$ spigots. Complete specifications for these hydrocyclones were given in previous reports. The samples were sieved into individual size fractions, and each size fraction was chemically analyzed to determine the iron content. From the iron analyses, the relative percentages of magnetite and quartz at each size was calculated. These values were then used to calculate hydrocyclone efficiency curves for (a) magnetite alone, (b) quartz alone, and (c) the overall mixture of both minerals. Since the magnetite specific gravity (5.2) is significantly higher than the quartz specific gravity (2.65), the cut size for the magnetite efficiency curve was finer than the cut size of the quartz efficiency curve, as can be seen in Figure 1.

The hydrocyclones were being operated in closed circuit with a grinding mill, so that all of the mill product was circulated back to the hydrocyclones. Since the magnetite had to 
be ground to a finer size than the quartz before it was removed from the circuit, the hydrocyclone feed therefore was considerably enriched in magnetite at the finer sizes. This resulted in the behavior of the overall efficiency curve seen in Figure 1, where the overall curve initially was very close to what would be expected if the magnetite and quartz curves were averaged, but then very closely followed just the magnetite curve at the sizes where the feed was highly enriched in magnetite.

Once these curves were calculated, they were initially modelled using Plitt's equation (Plitt, 1976):

$$
\begin{aligned}
& y^{\prime}=1-\exp \left(-0.693\left(d / d_{50 c}\right)^{m}\right) \\
& d_{50 c}=\frac{C_{1} D^{0.46} D_{A}^{0.6} D_{O}^{1.21} \exp (0.063 \phi)}{D_{U}^{0.71} H^{0.38} Q_{V}^{0.45}\left(\rho_{m}-\rho_{l}\right)^{K_{2}}} \\
& m=1.93 \exp \left(-1.58 R_{V}\left(\frac{D^{2} H}{Q_{V}}\right)^{0.15}\right.
\end{aligned}
$$

where: $y^{\prime}=$ Corrected partition coefficient

$\mathrm{d}=$ Particle diameter (microns)

$\mathrm{d}_{50 \mathrm{c}}=$ Corrected cut size (microns)

$\mathrm{m}=$ Sharpness of separation

$\mathrm{D}=$ Cyclone diameter $(\mathrm{cm})$

$\mathrm{D}_{\mathrm{A}}=$ Feed inlet diameter $(\mathrm{cm})$

$\mathrm{D}_{\mathrm{O}}=$ Vortex finder diameter $(\mathrm{cm})$

$\phi=$ Solids fraction by volume in the cyclone feed

$\mathrm{D}_{\mathrm{U}}=$ Apex diameter $(\mathrm{cm})$

$\mathrm{H}=$ Distance between overflow and underflow $(\mathrm{cm})$

$\mathrm{Q}_{\mathrm{V}}=$ Volumetric flow rate of the feed pulp (liters/min)

$\rho_{\mathrm{m}}=$ specific gravity of solid particles

$\rho_{1}=$ specific gravity of liquid

$\mathrm{R}_{\mathrm{v}}=$ Fraction of the volumetric flowrate reporting to the underflow

$\mathrm{C}_{1}=$ Dimensional constant (equal to 50.5 for metric units)

$\mathrm{K}_{2}=$ Density exponent (assumed to equal 0.5 by all previous investigators)

\section{Results and Discussion}

The results of applying Plitt's model to the experimental hydrocyclone data are shown in Figure 1, where it can be immediately seen that the fit is very poor. This is primarily due to the distorted shape of the experimental curves compared to the smooth curves generated by Plitt's model. After extensive investigation, it was found that an alternative method could potentially be used that could adjust the shape of the model curve to match what was actually observed (Whiten, 2004): 
$y^{\prime}=\frac{(c(1+b x))(\exp (\alpha x)-1)}{\exp (\alpha x)+\exp (\alpha)-2}$

where: $\alpha=1.54 \mathrm{~m}-0.47$ (sharpness of separation)

$\mathrm{x}=\mathrm{d} / \mathrm{d}_{50 \mathrm{c}}$

$\mathrm{b}, \mathrm{c}=$ shape modification constants (reduces to standard shape when $\mathrm{b}=0, \mathrm{c}=1$ )

Least-squares analysis showed that, when the values $b=0.0021$ and $c=0.9712$ were used in the model, the fit to the observed data was greatly improved as shown in Figure 2, particularly for the gangue (quartz) curve. However, it was also determined that the model curves were now more closely spaced than the actual experimental curves, indicating that the model's prediction of the effects of specific gravity on the cut size were in error. This had never been noted by previous investigators because a finely ground ore with approximately equal quantities of high-density and low-density particles had never been subjected to this type of analysis before.

Examination of the deviation between the model results and the experimental data indicates that there are two possible reasons for the model underestimating the effect of particle density on the cut size:

1. The assumption that the exponent $\mathrm{K}_{2}=0.5$ may be incorrect. This exponent was assumed by past investigators to be equal to the value that would be expected from Stokes' Law, which is only valid for laminar flow. Since the flowrates in the hydrocyclone give Reynolds number values well above the normal laminar/turbulent transition, it is quite likely that this assumption is incorrect and the exponent may be anywhere from 0.5 (laminar) to 1.0 (fully turbulent).

2. The bulk density of the slurry in the hydrocyclone may vary markedly due to the changing concentrations of solids with position. This would result in marked increase in the buoyancy of the particles in the liquid, enhancing the difference in behavior between the high-density particles and the low-density particles. This effect is expected to be strongest for slurries that contain very fine particles, since coarse particles will behave as if the fine particles were part of a continuous medium rather than discrete particles.

Work is underway to determine the relative importance of these two factors on hydrocyclone performance.

\section{Conclusions}

In order to address the overgrinding caused by the "fish-hook" behavior of the hydrocyclones, it is necessary to remove the intermediate-sized magnetite that the hydrocyclones otherwise preferentially return to the grinding mills. In order for a model to be useful in determining methods for accomplishing this, it must accurately predict the behavior of hydrocyclones for this situation. 
It has been shown that the presence of different density particles results in a distortion of the efficiency curves that cannot be adequately matched using standard hydrocyclone modeling assumptions. A correction for the shape of the curve has been incorporated into the model which allows a much improved fit to the experimental data, by including two additional adjustable parameters.

Results with the modified model indicate for the first time that the previously accepted assumption that cyclone cut size is inversely proportional to $\left(\rho_{\text {solid }}-\rho_{\text {liquid }}\right)^{0.5}$ is, in fact, incorrect. Since validation tests of the model showed that the actual difference in performance due to density was significantly different from what was predicted by assuming that the value was equal to 0.5 , it is obvious that all models to date have been oversimplified on this point. The model is therefore being further modified and improved based on the validation data collected from the plant, to determine whether this is due to deviations of the exponent from the value of 0.5 , or whether it is a result of the bulk slurry density varying within the cyclone as the solids concentration changes..

The improved model will determine the actual dependence of cyclone performance on particle density, which is necessary to accurately model and control the quantity of highdensity material that is misplaced into the hydrocyclone coarse product and subsequently overground in the grinding mill.

\section{References}

L.G. Austin, R.R. Klimpel, P.T. Luckie. 1984. Process Engineering of Size Reduction: Ball Milling. Society of Mining Engineering, AIME. New York

A.R. Laplante and J.A. Finch, 1984. The Origin of Unusual Cyclone Performance Curves. International Journal of Mineral Processing, Volume 13, p 1 - p 11.

T.J. Napier-Munn, S. Morrel, R.D. Morrison, T. Kojovis. 2001. Mineral Comminution Circuits, Their Operation and Optimization. JKMRC Monograph Series in Mining and Mineral Processing 2. Australia.

L. R. Plitt, 1976, “A Mathematical Model of the Hydrocyclone Classifier”, CIM Bulletin, December, pp. 114-123

W. J.Whiten, 2004, Personal Communication, March 15

SSI, 2003. Systat Version 10.2, Systat Software Inc., <http://www.systat.com> 